Pacific Journal of Mathematics

ON MEROMORPHIC STARLIKE FUNCTIONS 


\section{ON MEROMORPHIC STARLIKE FUNCTIONS}

\section{Ch. Pommerenke}

1. Introduction. Let $\subseteq(\alpha)(0 \leqq \alpha<1)$ denote the class of functions of the form

$$
f(z)=z+\sum_{n=0}^{\infty} a_{n} z^{-n}
$$

that are analytic in $1<|z|<\infty$ and satisfy

$$
\text { Re } z \frac{f^{\prime}(z)}{f(z)} \geqq \alpha .
$$

The class $\mathfrak{S}(0)$ is formed by the meromorphic starlike functions $f(z)=$ $z+\cdots$, that is by the functions that map $\{|z|>1\}$ onto a region whose compact complement is starlike with respect to the origin.

Apart from the case $\alpha=0$ the most interesting case is $\alpha=1 / 2$. We shall see (Corollary 1) that every $f \in \mathcal{S}\left(\frac{1}{2}\right)$ can be approximated by the roots of polynomials with zeros in the unit disk, that is by functions of the form

$$
f(z)=\left(\prod_{\nu=1}^{n}\left(z-z_{\nu}\right)\right)^{1 / n} \quad\left(\left|z_{\nu}\right| \leqq 1\right) .
$$

These functions belong to $\mathfrak{S}\left(\frac{1}{2}\right)$.

The main results will be: $A s \quad r \rightarrow 1$,

$$
\max _{f \in \subseteq(\alpha)} \max _{|z| \leqq r}\left|f^{\prime}(z)\right| \sim \frac{2^{2(1-\alpha)} e^{-1}}{\left(1-r^{-1}\right) \log 1 /\left(1-r^{-1}\right)} .
$$

If $f \in \mathfrak{S}(\alpha)$ then

$$
\begin{aligned}
\left|f^{\prime}(z)\right| & \geqq \alpha\left|z^{-1} f(z)\right|+(1-\alpha)\left|z^{-1} f(z)\right|^{-\alpha /(1-\alpha)}\left(1-|z|^{-2}\right) \\
& \geqq\left(1-|z|^{-2}\right)^{1-\alpha} .
\end{aligned}
$$

For each fixed $f \in \mathfrak{S}(\alpha)$,

$$
\left|f^{\prime}(z)\right| \geqq \kappa\left(1-|z|^{-1}\right)^{\max (1-2 \alpha, \varepsilon)}
$$

for every $\varepsilon>0$ and some constant $\kappa=\kappa(f, \varepsilon)>0$.

The coefficients of $f(z)$ satisfy

$$
\left|a_{n}\right| \leqq \frac{2(1-\alpha)}{n+1}
$$

Received August 8, 1962. Research supported by National Science Foundation under grant G 18837. 


$$
\underset{n \rightarrow \infty}{\inf \sup _{n \rightarrow \infty} n\left|a_{n}\right|<2(1-\alpha) .}
$$

All these inequalities are best possible.

Inequality (1.3) is well-known for the case $\alpha=0$ whereas (1.2) seems to be new even for $\alpha=0$. Inequality (1.4) has been proved by Clunie [1] for $\alpha=0$. Some of the problems can also be studied by the variation method developed by Royster [9].

We shall also prove inequalities analogous to (1.4) and (1.5) for functions $g(\zeta)$ that are analytic and bounded in $|\zeta|<1$ and satisfy $\operatorname{Re} \zeta g^{\prime}(\zeta) / g(\zeta) \geqq \alpha$. (For $\alpha=0$, see [2] and [7]).

\section{A representation formula.}

Theorem 1. Let $f \in \mathfrak{S}(\alpha)(0 \leqq \alpha<1)$. Then

$$
f(z)=z \exp \left(2(1-\alpha) \int_{0}^{2 \pi} \log \left(1-e^{i t} z^{-1}\right) d \gamma(t)\right)
$$

where $\gamma(t)$ increases and $\gamma(t+2 \pi)-\gamma(t)=1$. Also $\arg f\left(r e^{i t}\right) \rightarrow \pi t+$ $2 \pi(1-\alpha) \gamma(t)$ as $r \rightarrow 1$, and

$$
z \frac{f^{\prime}(z)}{f(z)}=\alpha+(1-\alpha) \int_{0}^{2 \pi} \frac{1+e^{i t} z^{-1}}{1-e^{i t} z^{-1}} d \gamma(t) .
$$

Conversely, every function of the form (2.1) belongs to $\mathfrak{S}(\alpha)$.

Proof. We shall reduce (2.1) in the general case to the case $\alpha=0$ where (2.1) is a known formula. If $f \in \mathfrak{S}(\alpha)$ let

$$
g(z)=z^{-\alpha /(1-\alpha)} f(z)^{1 /(1-\alpha)}=z+\cdots .
$$

Then $\operatorname{Re} g^{\prime}(z) / g(z)=-\alpha /(1-\alpha)+1 /(1-\alpha) \cdot \operatorname{Re}_{z} f^{\prime}(z) / f(z) \geqq 0$. Hence $g(z)$ is starlike and (compare [7, Lemma 1])

$$
\begin{gathered}
g(z)=z \exp \left(\frac{1}{\pi} \int_{0}^{2 \pi} \log \left(1-e^{i t} z^{-1}\right) d V(t)\right), \\
z g^{\prime}(z) / g(z)=\frac{1}{2 \pi} \int_{0}^{2 \pi}\left(1+e^{i t} z^{-1}\right) /\left(1-e^{i t} z^{-1}\right) d V(t)
\end{gathered}
$$

where $V(t)=\lim _{r \rightarrow 1} \arg g\left(r e^{i t}\right)$ is monotone increasing and $V(t+2 \pi)-$ $V(t)=2 \pi$. Putting $\gamma(t)=V(t) /(2 \pi)$ we see that $f(z)=z^{\alpha} g(z)^{1-\alpha}$ satisfies (2.1) and (2.2). Direct computation shows that every function of the form (2.1) belongs to $\subseteq(\alpha)$.

CoROLlaRY 1. For given $f \in \mathfrak{S}(\alpha)$, there is a sequence of functions $f_{n} \in \mathfrak{S}(\alpha)$ of the form

$$
f_{n}(z)=z \prod_{1}^{n}\left(1-z_{\nu} z^{-1}\right)^{2(1-\alpha) / n}
$$


that converges to $f(z)$ locally uniformly in $|z|>1$. All functions of this form belong to $\subseteq(\alpha)$.

This corollary follows immediately from (2.1) by approximating $\gamma(t)$ by step-functions with $n$ steps of height $1 / n$. The last assertion of the corollary is established by computation. For $\alpha=\frac{1}{2}$, the corollary shows that $f$ can be approximated by functions of the form (1.1).

\section{Estimates of the function-values.}

THEOREM 2. Let $f(z)=z+a_{0}+a_{1} z^{-1}+\cdots \in \subseteq(\alpha)$ and $|z|=r>1$. Then

$$
\begin{aligned}
& \left|\frac{f(z)}{z}\right| \leqq\left(1+\frac{\left|a_{0}\right|}{1-\alpha} r^{-1}+r^{-2}\right)^{1-\alpha} \leqq\left(1+r^{-1}\right)^{2(1-\alpha)}, \\
& \left|\frac{f(z)}{z}\right| \geqq\left(1-r^{-1}\right)^{1-\alpha+\left|a_{0}\right| 1 / 2}\left(1+r^{-1}\right)^{1-\alpha-\left|a_{0}\right| 1 / 2} \geqq\left(1-r^{-1}\right)^{2(1-\alpha)} .
\end{aligned}
$$

Equality can be attained in all inequalities.

For $\alpha=0$ we find $\left(1+r^{-1}\right)^{2} \geqq\left|z^{-1} f(z)\right| \geqq\left(1-r^{-1}\right)^{2}$, and these are well-known inequalities which hold for all functions $f(z)=z+\cdots$ univalent and $\neq 0$ in $|z|>1$.

Proof of (3.1). Using (2.1) and the fact that the geometric mean is not greater than the arithmetic mean we see that

$$
\begin{aligned}
\left|z^{-1} f(z)\right|^{1 /(1-\alpha)} & =\exp \left(\int_{0}^{2 \pi} \log \left|1-e^{i t} z^{-1}\right|^{2} d \gamma(t)\right) \\
& \leqq \int_{0}^{2 \pi}\left|1-e^{i t} z^{-1}\right|^{2} d \gamma(t) \\
& =\int_{0}^{2 \pi}\left(1+r^{-2}\right) d \gamma(t)-2 R e\left[z^{-1} \int_{0}^{2 \pi} e^{-i t} d \gamma(t)\right] .
\end{aligned}
$$

Also by (2.1)

$$
a_{0}=-2(1-\alpha) \int_{0}^{2 \pi} e^{i t} d \gamma(t)
$$

Hence $\left|a_{0}\right| \leqq 2(1-\alpha)$, and by (3.3)

$$
\left|z^{-1} f(z)\right|^{1 /(1-\alpha)} \leqq 1+r^{-2}+\left|a_{0}\right| r^{-1} /(1-\alpha) \leqq\left(1+r^{-1}\right)^{2}
$$

from which inequalities (3.1) follow. We have equality for the function

$$
f(z)=z\left(1+\frac{a_{0}}{1-\alpha} z^{-1}+z^{-2}\right)^{1-\alpha}=z+a_{0}+\cdots \in \mathfrak{S}(\alpha)
$$

where $0 \leqq a_{0} \leqq 2(1-\alpha)$, and $z=r>1$. 
Proof of (3.2). Since $z f^{\prime}(z) / f(z)=1-a_{0} z^{-1}+\cdots$ and $\operatorname{Re} z f^{\prime}(z) / f(z) \geqq$ $\alpha$ the function

$$
\phi(z)=z \frac{z f^{\prime}(z) / f(z)-1}{z f^{\prime}(z) / f(z)+(1-2 \alpha)}=-\frac{a_{0}}{2(1-\alpha)}+\cdots
$$

is analytic in $|z|>1$ and satisfies $|\phi(z)|<1$. Then

(3.5) $\frac{\partial}{\partial r} \log \left|z^{-1} f(z)\right|=-\frac{1}{r}+\operatorname{Re}\left[\frac{z}{r} \frac{f^{\prime}(z)}{f(z)}\right] \leqq 2(1-\alpha)\left|\frac{z^{-1} \phi(z)}{r\left(1-z^{-1} \phi(z)\right)}\right| \cdot$

If $b=|\phi(\infty)|=\left|a_{0}\right| / 2(1-\alpha)$ then [3, p. 287]

$$
|\phi(z)| \leqq(b r+1) /(b+r)
$$

hence by (3.5)

$$
\frac{\partial}{\partial r} \log \left|z^{-1} f(z)\right| \leqq 2(1-\alpha) \frac{b r+1}{r\left(r^{2}-1\right)} .
$$

Integration over $[r,+\infty]$ gives

$\log \left|z^{-1} f(z)\right| \geqq(1-\alpha)(1+b) \log \left(1-r^{-1}\right)+(1-\alpha)(1-b) \log \left(1+r^{-1}\right)$,

and inequalities (3.2) follow because $b=\left|a_{0}\right| / 2(1-\alpha)=|\phi(\infty)| \leqq 1$.

Equality is attained for $z=r>1$ by the function

$$
f(z)=z\left(1-z^{-1}\right)^{1-\alpha+a_{0} / 2}\left(1+z^{-1}\right)^{1-\alpha-a_{0} / 2}=z-a_{0}+\cdots
$$

with $0 \leqq a_{0} \leqq 2(1-\alpha)$.

We could have proved (3.1) by the same method as (3.2). But the proof directly from the representation formula seemed to be more interesting.

4. Upper estimate of the derivative. The best possible upper estimate of the derivative for the functions $f(z)=z+\cdots$ that are meromorphic and univalent in $|z|>1$ is $\left|f^{\prime}\left(r e^{i \theta}\right)\right| \leqq 1 /\left(1-r^{-2}\right)$ (see for instance [3, p. 120]). We shall show that this inequality can be improved for starlike functions and small $r>1$.

TheOREm 3. Let $0 \leqq \alpha<1$. Then as $r \rightarrow 1$

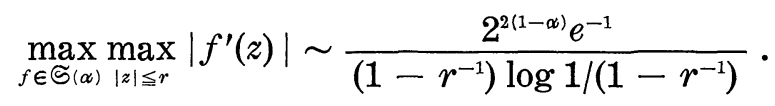

The proof will show that the function $f(z)$ for which $\left|f^{\prime}\left(z_{0}\right)\right|$ becomes maximal for a given $z_{0}$ has the form

$$
f(z)=z\left(1-z_{1} z^{-1}\right)^{2(1-\alpha) \gamma_{1}}\left(1-z_{2} z^{-1}\right)^{2(1-\alpha) \gamma_{2}}
$$


with $0 \leqq \gamma_{1} \leqq 1, \gamma_{1}+\gamma_{2}=1$ and $\left|z_{1}\right|=\left|z_{2}\right|=1$.

Proof. 1. Let $n \geqq 2$ and different $z_{\nu}(\nu=1, \cdots, n)$ with $\left|z_{\nu}\right|=1$ be given. We consider the functions

$$
f(z)=z \prod_{\nu=1}^{n}\left(1-z_{\nu} z^{-1}\right)^{2(1-\alpha) \gamma_{\nu}}
$$

with $0 \leqq \gamma_{\nu} \leqq 1, \gamma_{1}+\cdots+\gamma_{n}=1$. For fixed $z_{0}\left(\left|z_{0}\right|>1\right)$ let $f(z)$ be the function of this form for which $\left|f^{\prime}\left(z_{0}\right)\right|$ becomes maximal. We shall show that only two of the $\gamma_{\nu}$ can be $\neq 0$ for this maximal function. We shall use an elementary variation method (compare [5]).

2. Suppose this were false, and $\gamma_{\nu}>0(\nu=1,2,3)$. If $\beta_{1}+\beta_{2}+$ $\beta_{3}=0, \beta_{4}=\cdots=\beta_{n}=0$ then $\gamma_{\nu}^{*}=\gamma_{\nu} \pm \delta \beta_{\nu} \geqq 0$ for sufficiently small $\delta>0$, and $\Sigma \gamma_{\nu}^{*}=1$. Let $f_{*}(z)$ be the function of the form (4.2) where $\gamma_{\nu}$ has been replaced by $\gamma_{\nu}^{*}$. Let $\zeta=z_{0}^{-1}$. Then

$$
\begin{aligned}
& \left|f_{*}^{\prime}\left(z_{0}\right)\right|^{2}=\left|z_{0} f_{*}^{\prime}\left(z_{0}\right) / f_{*}\left(z_{0}\right)\right|^{2} \cdot\left|z_{0}^{-1} f_{*}\left(z_{0}\right)\right|^{2} \\
& \quad=\left|\sum_{\nu=1}^{n}\left(\gamma_{\nu} \pm \delta \beta_{\nu}\right) \frac{1+(1-2 \alpha) z_{\nu} \zeta}{1-z_{\nu} \zeta}\right|_{\nu=1}^{2} \prod_{\nu=1}^{n}\left|1-z_{\nu} \zeta\right|^{4(1-\alpha)\left(\gamma_{\nu} \pm \delta \beta_{\nu}\right)} .
\end{aligned}
$$

For abbreviation let

$$
c=\sum_{\nu=1}^{n} \gamma_{\nu} \frac{1+(1-2 \alpha) z_{\nu} \zeta}{1-z_{\nu} \zeta}, \quad b=\sum_{\nu=1}^{3} \beta_{\nu} \frac{1+(1-2 \alpha) z_{\nu} \zeta}{1-z_{\nu} \zeta}
$$

and

$$
l=\sum_{\nu=1}^{3} \beta_{\nu} \log \left|1-z_{\nu} \zeta\right| \text {. }
$$

Since $\beta_{4}=\cdots=\beta_{n}=0$ it follows that

$$
\begin{aligned}
\left|f_{*}^{\prime}\left(z_{0}\right)\right|^{2}= & \left(|c|^{2} \pm 2 \delta \operatorname{Re}[b \bar{c}]+\delta^{2}|b|^{2}\right) \cdot\left|z_{0}^{-1} f\left(z_{0}\right)\right|^{2} \\
& \cdot\left(1 \pm 4 \delta(1-\alpha) l+8 \delta^{2}(1-\alpha)^{2} l^{2}+0\left(\delta^{3}\right)\right) \\
= & \left|z_{0}^{-1} f\left(z_{0}\right)\right|^{2}\left[|c|^{2} \pm 2 \delta\left(\operatorname{Re}[b \bar{c}]+2(1-\alpha)|c|^{2} l\right)\right. \\
& \left.+\delta^{2}\left(|b|^{2}+8(1-\alpha) l \operatorname{Re}[b \bar{c}]+8(1-\alpha)^{2}|c|^{2} l^{2}\right)+0\left(\delta^{3}\right)\right] .
\end{aligned}
$$

Since $\left|f_{*}^{\prime}\left(z_{0}\right)\right|^{2} \leqq\left|f^{\prime}\left(z_{0}\right)\right|^{2}=\left|z_{0}^{-1} f\left(z_{0}\right)\right|^{2}|c|^{2}$ by the maximal choice of $f$, it follows that

$$
R e[b \bar{c}]+2(1-\alpha)|c|^{2} l=0
$$

and

$$
|b|^{2}+8(1-\alpha) l \operatorname{Re}[b \bar{c}]+8(1-\alpha)^{2}|c|^{2} l^{2} \leqq 0 .
$$

Eliminating $\operatorname{Re}[b \bar{c}]$ we obtain 


$$
|b|^{2} \leqq 8(1-\alpha)|c|^{2} l^{2}
$$

In particular, $l=0$ implies $b=0$.

The two homogeneous linear equations

$$
\begin{gathered}
l=\sum_{1}^{3} \beta_{\nu} \log \left|1-z_{\nu} \zeta\right|=0 \\
\beta_{1}+\beta_{2}+\beta_{3}=0
\end{gathered}
$$

have a nontrivial solution $\beta_{1}, \beta_{2}, \beta_{3}$. With these values it follows, as we have proved, that

$$
b=\sum_{1}^{3} \beta_{\nu} \frac{1+(1-2 \alpha) z_{\nu} \zeta}{1-z_{\nu} \zeta}=0 .
$$

Hence the three different points $w_{\nu}=\left(1+(1-2 \alpha) z_{\nu} \zeta\right) /\left(1-z_{\nu} \zeta\right)(\nu=$ $1,2,3)$ lie on a straight line, which is impossible because $\left|z_{\nu}\right|=1$ and $|\zeta|<1$. We have therefore proved that in the maximal function all exponents $\gamma_{\nu}$ are 0 except possibly two.

3. We can write this maximal function in the form (4.1). Thus, by (2.1) the function $f \in \mathfrak{S}(\alpha)$ for which $\left|f^{\prime}\left(z_{0}\right)\right|$ is maximal also has the form (4.1).

We may therefore assume that $f(z)$ has the form (4.1) and also that $\gamma_{1} \leqq \gamma_{2}$, hence $\gamma_{1} \leqq \frac{1}{2} \leqq \gamma_{2}$. Let $\rho=|z|^{-1}$. Then

$$
\begin{aligned}
\left|f^{\prime}(z)\right| \leqq & 2^{2(1-\alpha)}+2(1-\alpha) \gamma_{1}(1-\rho)^{2(1-\alpha) \gamma_{1}-1} 2^{2(1-\alpha) \gamma_{2}} \\
& +2(1-\alpha) \gamma_{2} \cdot 2^{2(1-\alpha) \gamma_{1}} \max \left[2^{2(1-\alpha) \gamma_{2}-1},(1-\rho)^{2(1-\alpha) \gamma_{2}-1}\right] .
\end{aligned}
$$

Since

$$
\max _{\gamma \geq 0} \gamma(1-\rho)^{2(1-\alpha) \gamma}=1 /[2(1-\alpha) e \log 1 /(1-\rho)]
$$

it follows that

$$
\left|f^{\prime}(z)\right| \leqq 4+2^{2(1-\alpha)} e^{-1}[(1-\rho) \log 1 /(1-\rho)]^{-1}+16(1-\rho)^{-\alpha} .
$$

4. We finally consider the special function

$$
f(z)=z\left(1-z^{-1}\right)^{2(1-\alpha) \gamma}\left(1+z^{-1}\right)^{2(1-\alpha)(1-\gamma)}
$$

with $\gamma=1 /[2(1-\alpha) \log 1 /(1-\rho)]$ for $\rho<1$ near to 1 . Then $f \in \mathfrak{S}(\alpha)$, and by (4.3)

$$
f^{\prime}\left(\rho^{-1}\right) \sim 2^{2(1-\alpha)} e^{-1}[(1-\rho) \log 1 /(1-\rho)]^{-1} \quad(\rho \rightarrow 1) .
$$

Together with (4.4) this proves Theorem 3. 
5. Lower estimates of the derivative. The best possible lower estimate of the derivative for functions $f(z)=z+\cdots$ meromorphic and univalent in $|z|>1$ is $\left|f^{\prime}(z)\right| \geqq 1-|z|^{-2}$, with equality for $f(z)=$ $z+z^{-1}$. This function belongs to $\subseteq(0)$. For $0<\alpha<1$ we can prove more.

TheOREM 4. If $f \in \mathfrak{S}(\alpha)(0 \leqq \alpha<1)$ then

$$
\left|f^{\prime}(z)\right| \geqq \alpha\left|\frac{f(z)}{z}\right|+(1-\alpha)\left|\frac{z}{f(z)}\right|^{\alpha /(1-\alpha)}\left(1-\frac{1}{|z|^{2}}\right) \geqq\left(1-\frac{1}{|z|^{2}}\right)^{1-\alpha} .
$$

Equality can be attained in all inequalities.

Proof. By (2.1) we have

$$
\left|\frac{z}{f(z)}\right|^{1 /(1-\alpha)}=\exp \left(\int_{0}^{2 \pi} \log \frac{1}{\left|1-e^{i t} z^{-1}\right|^{2}} d \gamma(t)\right)
$$

with $d \gamma(t) \geqq 0$ and $\int d \gamma(t)=1$. Since the geometric mean is not greater than the arithmetic mean it follows that

$$
\left|\frac{z}{f(z)}\right|^{1 /(1-\alpha)} \leqq \int_{0}^{2 \pi} \frac{1}{\left|1-e^{i t} z^{-1}\right|^{2}} d \gamma(t)
$$

Hence by (2.2)

$$
\begin{aligned}
\left|\frac{z}{f(z)}\right|^{1 /(1-\alpha)}\left(1-|z|^{-2}\right) & \leqq \int_{0}^{2 \pi} \frac{1-|z|^{-2}}{\left|1-e^{i t} z^{-1}\right|^{2}} d \gamma(t) \\
& =\frac{1}{1-\alpha}\left(\operatorname{Re} z \frac{f^{\prime}(z)}{f(z)}-\alpha\right)
\end{aligned}
$$

and therefore

$$
\left|z \frac{f^{\prime}(z)}{f(z)}\right| \geqq \operatorname{Re} z \frac{f^{\prime}(z)}{f(z)} \geqq \alpha+(1-\alpha)\left|\frac{z}{f(z)}\right|^{1 /(1-\alpha)}\left(1-|z|^{-2}\right)
$$

from which (5.1) follows. Equality is attained by

$$
f(z)=z\left(1-2 r^{-1} z^{-1}+z^{-2}\right)^{1-\alpha} \in \subseteq(\alpha)
$$

for $z=r>1$.

Though (5.1) is best possible for the whole class $\subseteq(\alpha)$ more is true for each fixed function in $\varsigma(\alpha)$ if $\alpha>0$.

THEOREM 5. Let $f \in \subseteq(\alpha)$. (i) If $0 \leqq \alpha<\frac{1}{2}$ then there is $a \kappa=$ $\kappa(f)>0$ such that

$$
\left|f^{\prime}\left(r e^{i \theta}\right)\right| \geqq \kappa\left(1-r^{-1}\right)^{1-2 \propto}
$$


The exponent cannot be decreased. (ii) If $\frac{1}{2} \leqq \alpha<1$ then

$$
\left|f^{\prime}\left(r e^{i \theta}\right)\right| \geqq \kappa(\varepsilon)\left(1-r^{-1}\right)^{\varepsilon}
$$

for every $\varepsilon>0$ where $\kappa(\varepsilon)=\kappa(f, \varepsilon)>0$.

Proof. We may take $\alpha>0$. Suppose Theorem 5 were false. Then there would be a sequence $z_{k}=r_{k} e^{i \theta_{k}}$ such that

$$
f^{\prime}\left(z_{k}\right)=o\left(\left(1-r_{k}^{-1}\right)^{1-2 \alpha}\right)
$$

and also

$$
f^{\prime}\left(z_{k}\right)=o\left(\left(1-r_{k}^{-1}\right)^{\varepsilon_{0}}\right)
$$

for some $\varepsilon_{0}>0$. We may assume that $\theta_{k} \rightarrow 0$. We have to distinguish two cases:

Case 1. The point $t=0$ is a discontinuity of the function $\gamma(t)$ of Theorem 1. Then let $\lambda_{0}$ be the height of the jump of $\gamma(t)$ at 0 , and let $\lambda_{j}(j=1, \cdots, m)$ be the jumps of height $\geqq \lambda_{0}$, occuring at $t_{j} \neq 0$. Let $\lambda^{*}$ be the highest jump $<\lambda_{0}$, and let $\delta=\lambda_{0}-\lambda^{*}$. Then $\delta>0$. If $\sigma(t)$ is the function that is constant except for jumps of height 1 at $0, \pm 2 \pi, \cdots$ let

$$
\gamma^{*}(t)=\gamma(t)-\lambda_{0} \sigma(t)-\sum_{j=1}^{m} \lambda_{j} \sigma\left(t-t_{j}\right) .
$$

This function increases and has highest jump $\lambda^{*}$. We see from Theorem 1 that

$$
\begin{aligned}
z_{k} \frac{f^{\prime}\left(z_{k}\right)}{f\left(z_{k}\right)}= & \alpha+(1-\alpha) \lambda_{0} \frac{1+z_{k}^{-1}}{1-z_{k}^{-1}}+(1-\alpha) \sum_{\mu=1}^{m} \lambda_{k} \frac{1+e^{i t} \mu z_{k}^{-1}}{1-e^{i t_{\mu}} z_{k}^{-1}} \\
& +F^{*}\left(z_{k}\right)
\end{aligned}
$$

where

$$
F^{*}(z)=(1-\alpha) \int_{0}^{2 \pi} \frac{1+e^{i t} z^{-1}}{1-e^{i t} z^{-1}} d \gamma^{*}(t)
$$

is a function of positive real part. Hence [4, Theorem 2]

$$
\left|F^{*}\left(z_{k}\right)\right| \leqq(1-\alpha)\left(\lambda^{*}+\frac{1}{3} \delta\right) /\left(1-r_{k}^{-1}\right)
$$

for large $k$. The third term on the right side of (5.5) is bounded as $k \rightarrow \infty$ because $\theta_{k} \rightarrow 0 \neq t_{j}(j=1, \cdots, m)$. Hence by (5.5) and (5.6)

$$
\begin{aligned}
\left|z_{k} f^{\prime}\left(z_{k}\right) / f\left(z_{k}\right)\right| & \geqq(1-\alpha)\left(\lambda_{0}-\frac{1}{3} \delta-\lambda^{*}-\frac{1}{3} \delta\right) /\left(1-r_{k}^{-1}\right) \\
& =\frac{1}{3} \delta /\left(1-r_{k}^{-1}\right)
\end{aligned}
$$


for large $k$. On the other hand, Theorem 2 implies $\left|z_{k}^{-1} f\left(z_{k}\right)\right| \geqq(1-$ $\left.r_{k}^{-1}\right)^{2-2 \alpha}$. Therefore $\left|f^{\prime}\left(z_{k}\right)\right| \geqq \frac{1}{3} \delta\left(1-r_{k}^{-1}\right)^{1-2 \alpha}$, in contradiction to (5.2).

Case 2. The function $\gamma(t)$ is continuous at $t=0$. Let now $\lambda_{j}(j=$ $1, \cdots, m)$ be the jumps of height $\geqq \varepsilon_{0} / 4(1-\alpha)$ occuring at $t_{j} \neq 0$. Let $\gamma^{*}(t)$ be defined as in (5.4) (with $\lambda_{0}=0$ ). Then we see from Theorem 1 that

$$
f(z)=\prod_{j=1}^{m}\left(1-e^{i t_{j}} z^{-1}\right)^{2(1-\alpha) \lambda_{j}} f^{*}(z)
$$

where

$$
f^{*}(z)=z \exp \left(2(1-\alpha) \int_{0}^{2 \pi} \log \left(1-e^{i t} z^{-1}\right) d \gamma^{*}(t)\right)
$$

is a starlike function. Hence $1 / f^{*}\left(\zeta^{-1}\right)$ is starlike in $|\zeta|<1$. Since all jumps of $\gamma^{*}(t)$ are $<\varepsilon_{0} / 4(1-\alpha)$ it follows [7, Theorem 1] that

$$
1 / f^{*}\left(\zeta^{-1}\right)=o\left((1-|\zeta|)^{-\varepsilon_{0}}\right) \text {. }
$$

Since $\theta_{k} \rightarrow 0 \neq t_{j}$ as $k \rightarrow \infty$ we therefore obtain from (5.7) that

$$
\left|z_{k}^{-1} f\left(z_{k}\right)\right| \geqq\left(1-r_{k}^{-1}\right)^{\varepsilon_{0}}
$$

for large $k$. Because $R e z f^{\prime}(z) / f(z) \geqq \alpha>0$ it follows that

$$
\left|f^{\prime}\left(z_{k}\right)\right| \geqq \alpha\left|z_{k}^{-1} f\left(z_{k}\right)\right| \geqq \alpha\left(1-r_{k}^{-1}\right)^{\varepsilon_{0}}
$$

in contradiction to (5.3).

Finally, that function $f(z)=z\left(1-z^{-1}\right)^{2-2 \alpha} \in \subseteq(\alpha)$ shows that $1-2 \alpha$ cannot be made smaller.

6. Estimates of the coefficients If $f(z)=z+a_{0}+a_{1} z^{-1}+\cdots$ is a starlike function then, as Clunie [1] has proved, $\left|a_{n}\right| \leqq 2 /(n+1)$. To generalise this inequality to the class $\subseteq(\alpha)$ we first prove the following lemma, using Clunie's method.

Lemma 1. If $f \in S(\alpha)(0 \leqq \alpha<1)$ and $n=0,1, \cdots$ then

$$
(n+1)^{2}\left|a_{n}\right|^{2} \leqq 4(1-\alpha)^{2}-4(1-\alpha) \sum_{\nu=0}^{n-1}(\nu+\alpha)\left|a_{\nu}\right|^{2} .
$$

Proof. As in (3.4) let again

$$
\phi(z)=z \frac{z f^{\prime}(z)-f(z)}{z f^{\prime}(z)+(1-2 \alpha) f(z)} .
$$

Then it follows that 


$$
-\sum_{\nu=0}^{\infty}(\nu+1) a_{\nu} z^{-\nu}=\phi(z)\left[2(1-\alpha)-\sum_{\nu=0}^{\infty}(\nu-1+2 \alpha) a_{\nu} z^{-\nu-1}\right],
$$

hence for $n=0,1, \cdots$

$$
\begin{gathered}
-\sum_{\nu=0}^{n}(\nu+1) a_{\nu} z^{-\nu}-\sum_{\nu=n+1}^{\infty}(\nu+1) a_{\nu} z^{-\nu}+\phi(z) \sum_{\nu=n}^{\infty}(\nu-1+2 \alpha) a_{\nu} z^{-\nu-1} \\
=\phi(z)\left[2(1-\alpha)-\sum_{\nu=0}^{n-1}(\nu-1+2 \alpha) a_{\nu} z^{-\nu-1}\right] .
\end{gathered}
$$

Since the second and third term on the left side involve only powers $z^{-\mu}$ with $\mu \geqq n+1$ and since $|\phi(z)|<1$ Parceval's formula gives

$$
\sum_{\nu=0}^{n}(\nu+1)^{2}\left|a_{\nu}\right|^{2} \leqq 4(1-\alpha)^{2}+\sum_{\nu=0}^{n-1}(\nu-1+2 \alpha)^{2}\left|a_{\nu}\right|^{2},
$$

and Lemma 1 follows at once.

CoROLlary 2. Let $f \in \mathfrak{S}(\alpha), 0<\alpha<1$, and let $A$ be the area of the compact complement of the image region $\{f(z):|z|>1\}$. Then

$$
\pi \geqq A>\pi \alpha,
$$

and these inequalities are best possible.

Proof. The inequality $\pi \geqq A$ is of course classical. Lemma 1 implies

$$
\sum_{\nu=0}^{\infty}(\nu+\alpha)\left|a_{\nu}\right|^{2} \leqq 1-\alpha,
$$

hence

$$
A=\pi\left(1-\sum_{\nu=1}^{\infty} \nu\left|a_{\nu}\right|^{2}\right) \geqq \pi\left(\alpha+\alpha \sum_{\nu=0}^{\infty}\left|a_{\nu}\right|^{2}\right) \geqq \pi \alpha .
$$

Equality could only hold if $a_{\nu}=0$ for $\nu=0,1, \ldots$. But then $A=\pi$.

To show that $A>\pi \alpha$ is best possible we consider

$$
f(z)=z\left(1+z^{-n-1}\right)^{2(1-\alpha) /(n+1)}=z+\frac{2(1-\alpha)}{n+1} z^{-n}+\cdots \in \mathfrak{S}(\alpha) .
$$

The function $w=f(z)$ maps $|z|=1$ onto a set contained in

$$
\begin{aligned}
\{|w| & \leqq 2^{2(1-\alpha) /(n+1)},|\arg w-2 \pi k /(n+1)| \\
& \leqq \pi \alpha /(n+1), k=0, \cdots, n\} .
\end{aligned}
$$

Therefore $A$ is smaller than the area $\pi \alpha \cdot 2^{4(1-\alpha) /(n+1)}$ of this last set which tends $\rightarrow \pi \alpha$ as $n \rightarrow \infty$.

THEOREM 6. Let $f(z)=z+\sum_{n=0}^{\infty} a_{n} z^{-n}$ be in $\subseteq(\alpha) \quad(0 \leqq \alpha<1)$. 
Then

$$
\left|a_{n}\right| \leqq \frac{2(1-\alpha)}{n+1}
$$

for $n=0,1, \cdots$, with equality for the functions (6.1).

Inequality (6.2) follows immediately from Lemma 1 . The next theorem will show that (6.2) cannot be improved much even for a fixed function in $\subseteq(\alpha)$. In particular, there is a starlike function with $a_{n} \neq o\left(n^{-1}\right)$.

TheOREM 7. If $f \in \subseteq(\alpha)$ then

$$
\limsup _{n \rightarrow \infty} n\left|a_{n}\right|<2(1-\alpha) \text {. }
$$

For every $\varepsilon>0$ there is a function $f \in \mathfrak{S}(\alpha)$ such that

$$
\limsup _{n \rightarrow \infty} n\left|a_{n}\right|>2(1-\alpha)-\varepsilon
$$

Proof. It follows from Lemma 1 that

$\limsup _{n \rightarrow \infty}(n+1)^{2}\left|a_{n}\right|^{2} \leqq 4(1-\alpha)^{2}-4(1-\alpha) \sum_{\nu=0}^{\infty}(\nu+\alpha)\left|a_{\nu}\right|^{2}<4(1-\alpha)^{2}$

except when $a_{\nu}=0$ for $\nu=0,1, \cdots$. But then (6.3) is trivial. The last assertion will be proved at the end of this paper.

We shall now consider bounded starlike functions that are analytic in $|\zeta|<1$.

THEOREM 8. Let $g(\zeta)=\sum_{n=1}^{\infty} b_{n} \zeta^{n}$ be analytic in $|\zeta|<1$ and satisfy

$$
\operatorname{Re} \zeta g^{\prime}(\zeta) / g(\zeta) \geqq \alpha .
$$

and $|g(\zeta)|<1$. Then

$$
\sum_{n=1}^{\infty}(n-\alpha)\left|b_{n}\right|^{2} \leqq 1-\alpha .
$$

For $n=2,3, \cdots$

$$
\left|b_{n}\right|<\frac{2(1-\alpha)}{n+1-2 \alpha} .
$$

The factor $2(1-\alpha)$ cannot be replaced by a smaller factor independent of $n$. For every $\varepsilon>0$ there is a function $g(\zeta)$ such that

$$
\limsup _{n \rightarrow \infty} n\left|b_{n}\right|>2(1-\alpha)-\varepsilon \text {. }
$$

In the case $\alpha=0$ inequality (6.6) has been proved (in a slightly 
weaker form) by Clunie and Keogh [2], and in [7, Theorem 3]. Clunie and Keogh also gave an example of a starlike bounded function with $b_{n} \neq o\left(n^{-1}\right)$.

Proof. For $0<\rho<1$

$$
\sum_{n=1}^{\infty}(n-\alpha)\left|b_{n}\right|^{2} \rho^{2 n}=\frac{1}{2 \pi} \int_{0}^{2 \pi}\left|g\left(\rho e^{i \theta}\right)\right|^{2}\left(\operatorname{Re}\left[\rho e^{i \theta} g^{\prime} \mid g\right]-\alpha\right) d \theta .
$$

Since $\left|g\left(\rho e^{i \theta}\right)\right|<1$ it follows by (6.4) that

$$
\sum_{n=1}^{\infty}(n-\alpha)\left|b_{n}\right|^{2} \rho^{2 n} \leqq \frac{1}{2 \pi} \int_{0}^{2 \pi}\left(\operatorname{Re}\left[\rho e^{i \theta} g^{\prime} / g\right]-\alpha\right) d \theta=1-\alpha
$$

which implies (6.5)

As in the proof of Lemma 1 we obtain

$$
(n+1-2 \alpha)^{2}\left|b_{n}\right|^{2} \leqq 4(1-\alpha) \sum_{\nu=1}^{n}(\nu-\alpha)\left|b_{\nu}\right|^{2} .
$$

By (6.5) this expression is $\leqq 4(1-\alpha)^{2}$. If we had equality it would follow from $(6.5)$ that $g(\zeta)=b_{1} \zeta+\cdots\left(b_{1} \neq 0\right)$ is a polynomial of degree $n>1$. Then $\zeta g^{\prime}(\zeta) / g(\zeta)$ is continuous and $|g(\zeta)|<1$ almost everywhere on $|\zeta|=1$, and (6.8) shows that (6.5) holds with strict inequality.

Let $g_{0}(\zeta)=n^{1 /(n-1)}\left(\zeta+2 n^{-1} \zeta^{n}+\cdots\right)$ be the starlike function of Example 1 in [7]. It satisfies $\left|g_{0}(\zeta)\right|<1$. Then

$$
g(\zeta)=\zeta^{\alpha} g_{0}(\zeta)^{1-\alpha}=n^{-(1-\alpha) /(n-1)}\left(\zeta+2(1-\alpha) n^{-1} \zeta^{n}+\cdots\right)
$$

satisfies (6.4) and $|g(\zeta)|<1$. Hence the factor $2(1-\alpha)$ in (6.6) cannot be made smaller. The existence of a function with (6.7) will be proved in the following.

7. Construction of examples. We shall now complete the proofs of Theorems 7 and 8 by showing: For every $\varepsilon>0$ there exist functions

$$
f(z)=z+\sum_{n=0}^{\infty} a_{n} z^{-n}
$$

in $\mathfrak{S}(\alpha)$ and

$$
g(\zeta)=\sum_{1}^{\infty} b_{n} \zeta^{n}
$$

with $\operatorname{Re} \zeta g^{\prime}(\zeta) / g(\zeta) \geqq \alpha$ and $|g(\zeta)|<1$ such that

$$
\limsup _{n \rightarrow \infty} n\left|a_{n}\right|>2(1-\alpha)-\varepsilon, \quad \limsup _{n \rightarrow \infty} n\left|b_{n}\right|>2(1-\alpha)-\varepsilon .
$$

We shall need the following lemma. 
LEMma 2. Given $\delta>0$ and $\eta>0$ there exists a function

$$
h(\zeta)=1+\sum_{n=1}^{\infty} c_{n} \zeta^{n}
$$

with $c_{n} \geqq 0$ that is analytic and has positive real part in $|\zeta|<1$ such that

$$
\begin{gathered}
\limsup _{n \rightarrow \infty} c_{n}>2-\delta, \\
\sum_{1}^{\infty} \frac{c_{n}}{n}<\eta .
\end{gathered}
$$

This lemma was first proved by F. Riesz [8] but only with limsup $c_{n} \geqq$ 1. It seems not to be known in the general form given here. It will be seen that the weaker form is not sufficient to prove the existence of an $f \in \mathfrak{S}(0)$ with $a_{n} \neq o\left(n^{-1}\right)$. But the weaker form is sufficient to prove the existence of a bounded starlike function $g(\zeta)$ with $b_{n} \neq o\left(n^{-1}\right)$ [2].

Proof. Let $1>\lambda>1-\frac{1}{2} \delta$ and let $p$ be so large that

$$
\begin{aligned}
P(\theta) & =1+2 \lambda \cos \theta+\cdots+2 \lambda^{p} \cos p \theta \\
& =\operatorname{Re}\left[1+2 \lambda e^{i \theta}+\cdots+2\left(\lambda e^{i \theta}\right)^{p}\right]>0 .
\end{aligned}
$$

Let $q$ be such that

$$
(2 p+1) 2^{-q}<\min \left(\frac{1}{8} \eta, \frac{1}{2}\right) .
$$

For $m=1,2, \cdots$ let

$$
Q_{m}(\theta)=\prod_{k=1}^{m} P\left(2^{q k} \theta\right)
$$

Then $Q_{m}(\theta)>0$. Because of (7.3) induction shows that

$$
Q_{m}(\theta)=1+\sum_{k=1}^{2 p \cdot 2 q m} c_{n} \cos n \theta
$$

where $c_{n}$ is independent of $m$ and $0 \leqq c_{n} \leqq 2, c_{2 q m}=2 \lambda$, and $c_{n}>0$ only if

$$
n=\Sigma \mu_{k} 2^{9 k} \quad\left(-p \leqq \mu_{k} \leqq p\right) .
$$

It follows that

$$
\sum_{1}^{\infty} \frac{c_{n}}{n} \leqq \Sigma^{\prime} \frac{2}{n}
$$

where the last sum is taken over all $n$ of the form (7.5). For $j=1,2, \cdots$, we group together all those $n$ for which $\mu_{j} \neq 0$ but $\mu_{k}=0$ for $k>j$. 
Then $n \geqq 2^{q j}-p 2^{q(j-1)}-\cdots-p 2^{q}>2^{q j}\left(1-2 p \cdot 2^{-q}\right)>2^{q j-1}$ by (7.3). The number of these $n$ is $<(2 p+1)^{j}$. Hence again by (7.3)

$$
\sum_{1}^{\infty} \frac{c_{n}}{n} \leqq 2 \sum_{j=1}^{\infty}(2 p+1)^{j} 2^{-q j+1}<8(2 p+1) 2^{-q}<\eta .
$$

The function

$$
h(\zeta)=1+\sum_{n=1}^{\infty} c_{n} \zeta^{n}
$$

is analytic in $|\zeta|<1$, and $\operatorname{Re} h(\zeta) \geqq 0$ by (7.4). Also $c_{2 q m}=2 \lambda>2-\delta$, and Lemma 2 is proved.

We shall now construct the starlike functions. Let $0<\varepsilon<1$ and $\delta=\varepsilon / 6, e^{(1-\alpha) \eta}=1+\varepsilon / 6$. If $h(\zeta)$ is the function of Lemma 2 let

$$
H(\zeta)=\int_{0}^{\zeta} s^{-1}(h(s)-1) d s=\sum_{n=1}^{\infty} \frac{c_{n}}{n} \zeta^{n} .
$$

Let $\beta=1-\alpha$ and

$$
f(z)=z \exp \left[-\beta H\left(z^{-1}\right)\right], \quad g(\zeta)=\zeta \exp [\beta(H(\zeta)-\eta)] .
$$

Then $z f^{\prime}(z) / f(z)=1+\beta z^{-1} H^{\prime}\left(z^{-1}\right)=\alpha+(1-\alpha) h\left(z^{-1}\right)$, hence $f(z)=$ $z+\cdots \in \subseteq(\alpha)$. Also, $g(\zeta)$ satisfies (6.4), and by (7.2)

$$
|g(\zeta)| \leqq \exp \left[\beta\left(\sum_{1}^{\infty} n^{-1} c_{n}-\eta\right)\right]<1
$$

By (7.6),

$$
\zeta^{-1} g(\zeta) e^{\beta \eta}-\zeta f\left(\zeta^{-1}\right)=2\left(\beta H(\zeta)+\frac{1}{3 !} \beta^{3} H(\zeta)^{3}+\cdots\right) .
$$

Since the coefficients $n^{-1} c_{n}$ of $H(\zeta)$ are nonnegative the function on the right side of (7.7) has coefficients $\geqq 2 \beta n^{-1} c_{n}$. Hence

$$
-a_{n-1}+b_{n+1} e^{\beta \eta} \geqq 2 \beta n^{-1} c_{n}
$$

and therefore

$$
\begin{aligned}
& n\left|a_{n-1}\right| \geqq 2 \beta c_{n}-n\left|b_{n+1}\right| e^{\beta \eta}, \\
& n\left|b_{n+1}\right| \geqq\left(2 \beta c_{n}-n\left|a_{n-1}\right|\right) e^{-\beta \eta} .
\end{aligned}
$$

By Theorems 6 and 8 we have $n\left|a_{n-1}\right| \leqq 2 \beta$ and $n\left|b_{n+1}\right| \leqq 2 \beta$. Therefore (7.8) and (7.9) together with (7.1) give

$$
\limsup _{n \rightarrow \infty} n\left|a_{n}\right| \geqq 2 \beta\left(2-\delta-e^{\beta \eta}\right)=2 \beta(1-\varepsilon / 3)>2 \beta-\varepsilon,
$$

$\limsup _{n \rightarrow \infty} n\left|b_{n}\right| \geqq 2 \beta(1-\delta) e^{-\beta \eta} \geqq 2 \beta(1-\varepsilon / 6) /(1+\varepsilon / 6)>2 \beta-\varepsilon$. 


\section{REFERENCES}

1. J. Clunie, On meromorphic schlicht functions, J. London Math. Soc., 34 (1959), 215-216.

2. J. Clunie and F. R. Keogh, On starlike and convex functions, J. London Math. Soc., 35 (1960), 229-233.

3. G. M. Golusin, Geometrische Funktionentheorie, Berlin 1957.

4. W. K. Hayman, On functions with positive real part, J. London Math. Soc., 36 (1961), 35-48.

5. G. Pólya and M. Schiffer, Sur la représentation conforme de l'extérieur d'une courbe fermée convexe, C. R. Acad. Sci. (Paris) 248 (1959), 2837-2839.

6. Ch. Pommerenke, Über einige Klassen meromorpher schlichter Funktionen, Math. Z., 78 (1962), 263-284.

7. - On starlike and convex functions, J. London Math. Soc., 37 (1962), 207-224.

8. F. Riesz, Über die Fourierkoeffizienten einer stetigen Funktion von beschränkter Schwankung, Math Z., 2 (1918), 312-315.

9. W. C. Royster, Extremal problems for functions starlike in the exterior of the unit circle, Canadian J. Math., 14 (1962), 540-551.

\section{CORNELL UNIVERSITY}





\section{PACIFIC JOURNAL OF MATHEMATICS}

\section{EDITORS}

\section{RalPh S. Phillips}

Stanford University

Stanford, California

M. G. Arsove

University of Washington

Seattle 5, Washington
J. Dugundji

University of Southern California Los Angeles 7, California

Lowell J. Paige

University of California

Los Angeles 24, California

\section{ASSOCIATE EDITORS}
E. F. BECKENBACH
D. DERRY
H. L. ROYDEN
E. G. STRAUS
T. M. CHERRY
M. OHTSUKA
E. SPANIER
F. WOLF

\section{SUPPORTING INSTITUTIONS}

\author{
UNIVERSITY OF BRITISH COLUMBIA \\ CALIFORNIA INSTITUTE OF TECHNOLOGY \\ UNIVERSITY OF CALIFORNIA \\ MONTANA STATE UNIVERSITY \\ UNIVERSITY OF NEVADA \\ NEW MEXICO STATE UNIVERSITY \\ OREGON STATE UNIVERSITY \\ UNIVERSITY OF OREGON \\ OSAKA UNIVERSITY \\ UNIVERSITY OF SOUTHERN CALIFORNIA
}

STANFORD UNIVERSITY

UNIVERSITY OF TOKYO

UNIVERSITY OF UTAH

WASHINGTON STATE UNIVERSITY

UNIVERSITY OF WASHINGTON

AMERICAN MATHEMATICAL SOCIETY

CALIFORNIA RESEARCH CORPORATION SPACE TECHNOLOGY LABORATORIES

NAVAL ORDNANCE TEST STATION

Printed in Japan by International Academic Printing Co., Ltd., Tokyo Japan 


\section{Pacific Journal of Mathematics}

\section{Vol. 13, No. 1 \\ March, 1963}

Frantz Woodrow Ashley, Jr., A cone of super-(L) functions............. 1

Earl Robert Berkson, Some metrics on the subspaces of a Banach space....

Felix Earl Browder and Walter Strauss, Scattering for non-linear wave

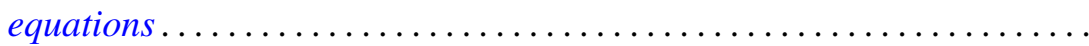

Edmond Darrell Cashwell and C. J. Everett, Formal power series ..........

Frank Sydney Cater, Continuous linear functionals on certain topological

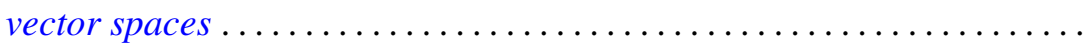

John Douglas Dixon, General group extensions ....................

Robert Pertsch Gilbert, On harmonic functions of four variables with

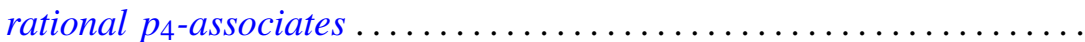

Irving Leonard Glicksberg, On convex hulls of translates ..............

Simon Hellerstein, On a class of meromorphic functions with deficient zeros

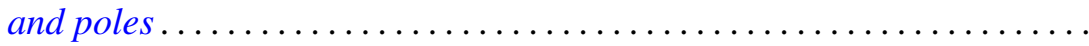

Donald William Kahn, Secondary cohomology operations which extend the

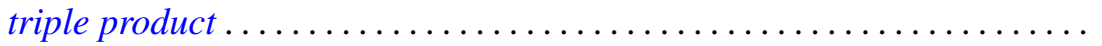

G. K. Leaf, A spectral theory for a class of linear operators .............

R. Sherman Lehman, Algebraic properties of the composition of solutions of partial differential equations ........................... 157

Joseph Lehner, On the generation of discontinuous groups ............. 169

S. P. Lloyd, On certain projections in spaces of continuous functions ...... 171 Fumi-Yuki Maeda, Generalized spectral operators on locally convex spaces ..................................

Donald Vern Meyer, $E^{3}$ modulo a 3-cell

William H. Mills, An application of linear programming to permutation groups.

Richard Scott Pierce, Centers of purity in abelian groups

Christian Pommerenke, On meromorphic starlike functions ...

Zalman Rubinstein, Analytic methods in the study of zeros of

polynomials...

B. N. Sahney, On the Nörlund summability of Fourier series

Tôru Saitô, Regular elements in an ordered semigroup . .

Lee Meyers Sonneborn, Level sets on spheres...........

Charles Andrew Swanson, Asymptotic estimates for limit point

problems .

Lucien Waelbroeck, On the analytic spectrum of Arens . .

Alvin (Murray) White, Singularities of a harmonic function of three

variables given by its series development .............

Kōichi Yamamoto, Decomposition fields of difference sets ...

Chung-Tao Yang, On the action of $\mathrm{SO}(3)$ on a cohomology manifold... 